\title{
Advanced Fluid Models for Multi-Parameter Condition Monitoring Systems for Lubricants and Hydraulic Fluids
}

\author{
Thomas Voglhuber-Brunnmaier ${ }^{1}$, Alexander O. Niedermayer ${ }^{2}$, \\ Friederich Feichtinger ${ }^{2}$, Bernhard Jakoby ${ }^{1}$ \\ 1 Johannes Kepler University, Altenbergerstr. 69, 4040 Linz, Austria \\ ${ }^{2}$ Micro Resonant Technologies GmbH, Franz-Kain-Weg 37, 4040 Linz, Austria \\ thomas.voglhuber-brunnmaier@jku.at
}

\begin{abstract}
Summary
A compact measurement unit for fluid monitoring based on simultaneous measurement of viscosity and density is introduced. It will be shown that a new fluid model allows to achieve higher accuracies, which is demonstrated by comparison to earlier models.
\end{abstract}

Keywords: viscosity, density, tuning fork, oil sensor

\section{Introduction}

In order to reduce maintenance costs as well as the risk of unplanned downtimes, industry gradually adopts online condition monitoring (OCM) methods combined with predictive or proactive maintenance approaches. Enabled by the increasing level of automation, plenty of data can be made available to maintenance personnel and condition monitoring algorithms, and sophisticated evaluation methods can be implemented to assist in planning of maintenance actions. With the implementation of such databased decision methods, the reliability and precision of the collected data obviously has significant impact on the effectiveness of the maintenance actions triggered. Furthermore, the sooner a problem can be identified, the easier and cheaper the appropriate maintenance action will be. So, in many cases, the benefit of a sensor increases over proportionately with its accuracy and long-term stability.

In this contribution, we present an innovative method to increase data reliability, as implemented in a novel fully automated online condition monitoring system for hydraulic fluids and lubricating oils. The device continuously monitors the viscosity, mass density, and several other relevant parameters of the fluid. With the integrated active temperature control, measurement data can be acquired at any desired reference temperature and thus are independent of the operating conditions of the machine. By cyclic variation of the temperature, additional information is provided and used for validating the consistency of the data.

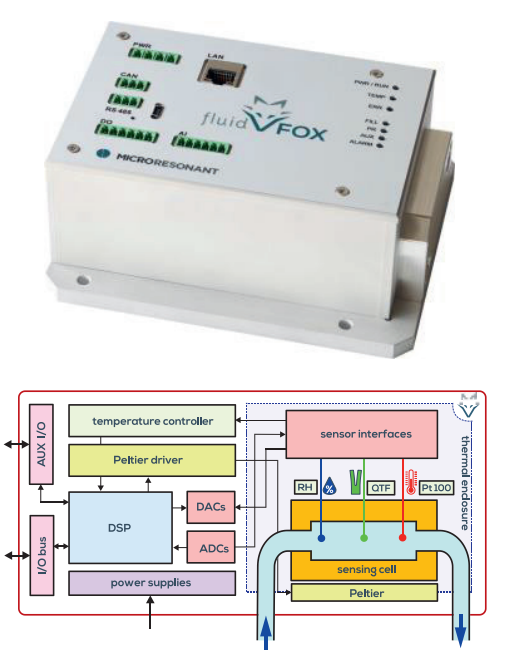

Fig. 1. Condition monitoring system for industrial use.

\section{Monitoring System}

Fig. 1 (above) shows the compact OCM system. The temperature-controlled measurement cell within the system houses the vibrating quartz tuning fork sensor (QTF), a Pt100 temperature sensor and a capacitive relative humidity sensor as shown in Fig. 1 (below). From the fluid induced resonance changes of the QTF the viscosity and density of the fluid are determined [1].

\section{Fluid Model}

The fluid models linking the resonance parameters $\left(f_{r}, Q\right)$ of a vibrating sensor to density $\rho$ and viscosity $\eta$ of the fluid are mostly based on truncated Taylor series approximations whose truncation is limited to an order of two to keep the inversion of the model manageable. The most accurate model of this class to date is the model of Heinisch [2] shown in eq. (1) featuring 6 instrument parameters $c_{1}$ to $c_{6}$. 


$$
\begin{aligned}
& f_{\mathrm{r}}=\left(c_{1}+c_{2} \rho+c_{3} \sqrt{\rho \eta / f_{\mathrm{r}}}\right)^{-\frac{1}{2}}, \\
& Q=\frac{1}{f_{\mathrm{r}}}\left(c_{4}+c_{5} \eta+c_{6} \sqrt{\rho \eta f_{\mathrm{r}}}\right)^{-1},
\end{aligned}
$$

We propose an alternative model shown in eq. (2), which allows for an arbitrary degree of approximation, not limited to second order.

$\underline{G}\left(f_{r}, Q\right)=\rho \sum_{i=0}^{N} \underline{c_{i}} \delta^{i}$

$$
\delta=\sqrt{\frac{\eta}{\pi f_{r} \rho}}
$$

We found an elegant new form where $\underline{G}$ represents a complex-valued function of the resonance parameters and $\underline{c_{i}}$ are complex-valued instrument parameters obtained by adjustment measurements using test fluids. $\delta$ is the characteristic decay length of plane shear waves. The inversion of the model is straight-forward and its complexity remains similar, when the order of approximation is extended to any arbitrary truncation $N$ of the Taylor series. Furthermore, new physical insights could be obtained using basic dimensional analysis [3]. It could be shown that this model is applicable for mechanical resonators of any shape, given that effects due to fluid compressibility and nonlinearity can be rendered negligible by design. The QTF sensor implemented in the fluidFOX in Fig. 1 is such a candidate.

Tab. 1: Table of the nominal values of the fluids measured using the fluidFOX. Deviations for viscosity are shown in Fig. 2

\begin{tabular}{ccccc} 
fluid \# & Fluid & $\begin{array}{r}\text { Temp. } \\
\left({ }^{\circ} \mathrm{C}\right)\end{array}$ & $\begin{array}{r}\text { Density } \\
\left(\mathbf{k g} / \mathrm{m}^{3}\right)\end{array}$ & $\begin{array}{c}\text { Dyn. visc. } \\
(\mathrm{mPas})\end{array}$ \\
\hline 1 & $\mathrm{~N} 2$ & 50 & 740.1 & 1.2760 \\
2 & ${ }^{*}$ N2 & 40 & 747.2 & 1.5060 \\
3 & $\mathrm{~N} 2$ & 25 & 757.8 & 1.9930 \\
4 & $\mathrm{~N} 2$ & 20 & 761.4 & 2.2120 \\
5 & $\mathrm{~N} 7$ & 50 & 781.1 & 4.4050 \\
6 & $\mathrm{~N} 7$ & 40 & 787.7 & 5.7680 \\
7 & $\mathrm{~N} 14$ & 50 & 793.3 & 8.2420 \\
8 & $\mathrm{~N} 7$ & 25 & 797.5 & 9.2720 \\
9 & $\mathrm{~N} 7$ & 20 & 800.8 & 11.110 \\
10 & $\mathrm{~N} 14$ & 40 & 799.7 & 11.290 \\
11 & $\mathrm{~N} 26$ & 50 & 801.9 & 14.350 \\
12 & $\mathrm{~N} 14$ & 25 & 809.3 & 19.670 \\
13 & $* \mathrm{~N} 26$ & 40 & 808.2 & 20.470 \\
14 & $\mathrm{~N} 44$ & 50 & 808.7 & 23.410 \\
15 & $\mathrm{~N} 14$ & 20 & 812.5 & 24.320 \\
16 & $\mathrm{~N} 44$ & 40 & 814.8 & 34.540 \\
17 & $\mathrm{~N} 26$ & 25 & 817.6 & 38.050 \\
18 & $\mathrm{~N} 26$ & 20 & 820.8 & 48.140 \\
19 & $* \mathrm{~N} 44$ & 25 & 824.1 & 68.120 \\
20 & $\mathrm{~N} 140$ & 50 & 819.1 & 71.950 \\
21 & $\mathrm{~N} 44$ & 20 & 827.2 & 87.860 \\
22 & $\mathrm{~N} 140$ & 40 & 825.1 & 112.20 \\
23 & $* \mathrm{~N} 140$ & 25 & 834.1 & 242.90 \\
\hline
\end{tabular}

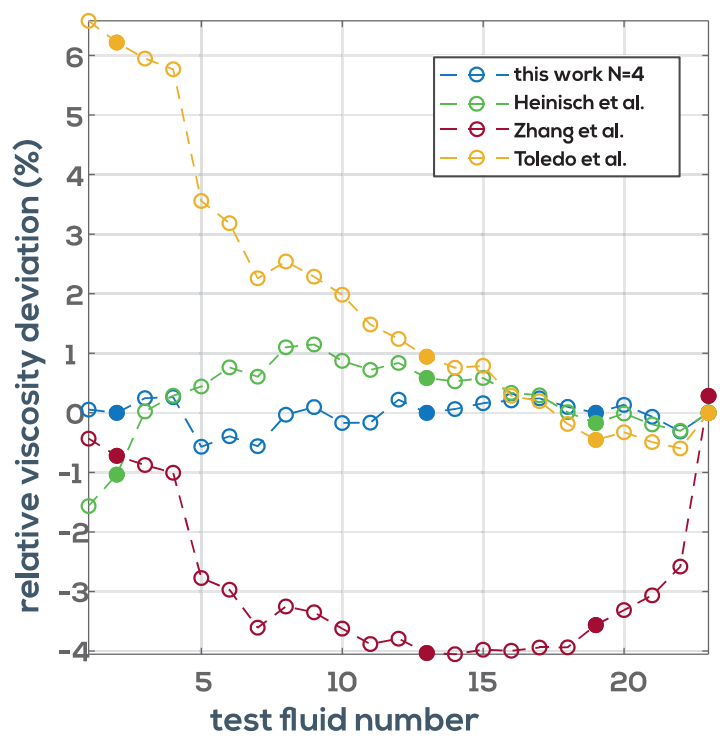

Fig. 2. Deviations between different fluid models. The viscosities of the fluid range from $1.27 \mathrm{mPas}$ to 242.9mPas. Fluids indicated by a filled marker correspond to fluids marked with * in Tab. 1 and were used to adjust the model parameters $c_{i}$ (see [5] for details).

We applied various models to the raw data available from the fluidFOX. Fig. 2 shows viscosity deviations where the new model used a truncation of $N=4$. The used respective NIST traceable reference fluids are listed in Tab. 1. The fluid marked with * were used to adjust the model parameters.

\section{Conclusions}

A new fluid model for simultaneous measuring of viscosity and density was established showing distinct advances over existing models.

\section{Acknowledgement}

This research was partly supported by Linz Center of Mechatronics (LCM) in the framework of the Austrian COMET-K2 programme.

\section{References}

[1] A.O. Niedermayer, T. Voglhuber-Brunnmaier, M. Heinisch, B. Jakoby, Accurate Determination of Viscosity and Mass Density of Fluids using a Piezoelectric Tuning Fork Resonant Sensor, Proceedings SENSOR 66-71. (2015), doi: 10.5162/sensor2015/A2.4

[2] M. Heinisch, T. Voglhuber-Brunnmaier, E.K. Reichel, I. Dufour, and B. Jakoby. (2014). Reduced order models for resonant viscosity and mass density sensors. Sensors and Actuators A: Physical, 220, 76-84.

[3] T. Voglhuber-Brunnmaier, and B. Jakoby (2020). Higher-order models for resonant viscosity and mass-density sensors. Sensors, 20(15), 4279. 